Journal of Applied Sciences, Engineering and Technology for Development Vol. 1, Issue1

Two-degree-of-freedom control of a multilink flexible manipulator using filtered inverse Feed-forward controller and strain feedback controller

\author{
Waweru Njeri $^{\mathrm{a}^{*}}$, Minoru Sasaki ${ }^{\mathrm{a}}$, Kojiro Matsushita ${ }^{\mathrm{a}}$, Harrison Ngetha \\ ${ }^{\mathrm{a}}$ Department of Mechanical Engineering, Faculty of Engineering, \\ Gifu University, 1-1 Yanagido, Gifu, 501-1193, Japan \\ ${ }^{\mathrm{b}}$ Department of Electrical \& Electronic Engineering, School of Engineering, \\ Dedan Kimathi University of Technology, P.O Box 657-10100, Nyeri, Kenya \\ *v3812104@edu.gifu-u.ac.jp
}

Manuscript history: Received: 17/05/2017; In revised form: 12/12/2018; Accepted: 11/02/2019 Published: 12/02/2019 


\title{
Two-degree-of-freedom control of a multilink flexible manipulator using filtered inverse feedforward controller and strain feedback controller
}

\begin{abstract}
This research paper presents a hybrid solution to both residue vibration and transient vibration as addressed using filtered inverse feedforward controller and strain feedback controller on a 3D 2 links flexible manipulator. The feedforward filtered inverse will minimize on the transient vibration due to sudden starting followed sudden stopping of the manipulator arm while the strain feedback will ensure rapid decay of the residue vibrations. Transient and residue vibrations stand in the way to reaping all the numerous advantages of the flexible manipulator. Transient vibration refers to vibrations resulting from sudden changes in the direction of the links while residue vibrations means that the arm takes too long to settle to do some useful work. Modelling of the manipulator and development of the inverse controller was carried out in Maple, MapleSim and MATLAB softwares. Experiments carried out in dSPACE control desk environment considering the manipulator without load and a case where a load of $100 \mathrm{~g}$ was attached at the distal end of link 2.
\end{abstract}

Keywords: Flexible manipulator, Transient vibration, Residue vibration, inverse controller, strain feedback 


\section{Waweru et al: Two-degree-of-freedom control of a multilink flexible manipulator using filtered inverse feedforward controller and strain feedback controller}

\section{INTRODUCTION}

Following the industrial revolution, robotic manipulators were designed to address some of the issues arising like working in hazardous conditions, precision, duplication, speed of operation etc. This bore the rigid manipulators which could be operated at high speeds and with high precision. Fabricated with heavy and bulky links, they were heavy and thus needed very big actuators thus consuming a lot of power. The solution came in form of flexible manipulators which are light in weight, cheap in cost and maintenance. However, flexible manipulators were to be operated at low speeds to avoid the excitation of transient vibration.

Flexible manipulators possess numerous strengths over rigid manipulators like light in weight, cheap in cost and maintenance to mention just but a few. To reap all these benefits of the flexible manipulator, it is important that main limitation they suffer from be ameliorated. This limitation is link vibration farther specified as transient vibration caused by sudden starts and sudden stops and residue vibration due to the shortcomings associated with the damping aspect of the system. Vibration is a menace as it leads to time wastage before the residue vibration can decay to levels where the manipulator can be put to useful work. It can also lead to Mechanisms failure due to vibrational fatigue. A case in point is that $30 \%$ of the operation time of the space robotic arm aboard the space station is wasted waiting for residue vibrations to decay to healthy levels before being put to useful work.

A lot of research has been carried out to mitigate link vibrations in a bid to reap all the benefits of the flexible manipulator. Authors in (Moheamani, 2003) made use of shunted piezoelectric transducers to mitigate vibrations. In this type of solution, piezoelectric transducers are bonded 
onto the links of the manipulator. Link flexure is converted to corresponding voltages which are used as feedback. The main limitation of this technique is that complexity of the feedback circuitry increases with increase in the number of links.

Low pass Finite Impulse Response (FIR) Filters have also been used in the suppression of residue vibrations. In this arrangement, the strain information is feedback though a digital filter which filters out the dominant modes of the link vibrations. Changes in loading which is followed by changes in the frequency spectrum of the flexure variable however renders this technique ineffective. This is addressed by using adaptive filters which can adapt to changes in loading.

In this paper, we explore the hybrid technique involving the inverse feedforward controller and the strain feedback control. Whereas open loop inverse control is effective for the mitigation of input related vibration (Alhazza, 2016), its performance on the residue vibration is not good. This way, it is supplemented with the closed loop strain feedback whose performance on the transient vibration is poor but very effective when it comes to residue vibration modes. Experiments are carried out considering the manipulator without any loading and loaded with a load of $100 \mathrm{~g}$.

This paper is organized as follows, in section 2, we present detailed theory on inversion of dynamic systems, in section 3, we apply the theory to invert a Multiple Input Multiple Output (MIMO) flexible manipulator followed by a brief theory of Direct Strain Feedback (DSFB) in section 4. Results are presented and discussed in section 5 and concluded in section 6. 


\section{Waweru et al: Two-degree-of-freedom control of a multilink flexible manipulator using filtered inverse feedforward controller and strain feedback controller}

\section{DEVELOPMENT OF THE INVERSE SYSTEM}

Consider a LTI continuous time square system $\Sigma$, let the triplet A, B and C be a minimal statespace representation. It is assumed that the system is stable or stabilized by negative feedback.

$$
\begin{array}{ll}
\dot{\mathrm{x}}(\mathrm{t}) & =\mathrm{Ax}(\mathrm{t})+\mathrm{Bu}(\mathrm{t}) \\
\mathrm{y}(\mathrm{t}) & =\mathrm{Cx}(\mathrm{t})
\end{array}
$$

Where $x(t) \in R^{n}, u(t) \in R^{p}, y=\left(y_{1}, y_{2}, \cdots, y_{p}\right)^{T} \in R^{p}, A \in R^{n \times n}, B \in R^{n \times p}$ and $C \in R^{p \times n}$. If $\mathrm{C}_{\mathrm{i}}$ denotes the $\mathrm{i}_{\text {th }}$ row of the output matrix $\mathrm{C}$, the system is said to have a well-defined relative degree $r \triangleq\left(r_{1}, r_{2}, \cdots r_{p}\right)^{T}$ if $C_{i} A^{l} B=0, \forall l<r_{i}-1,1 \leq i \leq p[15]$.

Differentiating $i_{\text {th }}$ output $r_{i}$ times in time yields

$$
y^{\left(r_{k}\right)}=C_{k} A^{\left(r_{k}\right)}+C_{k} A^{\left(r_{k}-1\right)} B u
$$

Where $\mathrm{C}_{\mathrm{k}}$ is the $\mathrm{k}_{\mathrm{th}}$ row of the output matrix $\mathrm{C}$ and $1<\mathrm{k}<\mathrm{p}$. In vector form

$$
y^{(r)}(t) \quad=\quad A_{x} x(t)+B_{y} u(t)
$$

Where

$$
\begin{aligned}
y^{(r)}(t) & \triangleq\left[y_{1}^{\left(r_{1}\right)}, y_{2}^{\left(r_{2}\right)}, \cdots, y_{p}^{\left(r_{p}\right)}\right]^{T} \\
B_{y} & \triangleq\left[C_{1} A^{\left(r_{1}\right)}, C_{1} A^{\left(r_{2}\right)}, \cdots, C_{1} A^{\left(r_{p}\right)}\right]^{T} \\
A_{x} & \triangleq\left[C_{1} A^{\left(r_{1}-1\right)}, C_{1} A^{\left(r_{2}-1\right)}, \cdots, C_{1} A^{\left(r_{p}-1\right)}\right.
\end{aligned}
$$

By is invertible because of the well-defined relative degree assumption. From equation (3), we can see that the control law is 


$$
u_{f f}=\mathrm{B}_{\mathrm{y}}^{-1}\left[\mathrm{y}_{\mathrm{d}}^{(\mathrm{r})}-\mathrm{A}_{\mathrm{x}}(\mathrm{t})\right] \quad \forall \mathrm{t} \in(-\infty, \infty)
$$

Then, there exist a state transformation $\mathrm{T}$

$$
[\xi(t) \eta(t)]=T x(t)
$$

Which transforms the states to internal dynamics $\eta(t)$ and the output and its derivatives in time up to $\left(\mathrm{r}_{1}-1\right)$ as

$$
\xi(t)=\left[y_{1}, \cdots, y_{1}^{\left(r_{1}-1\right)}, \cdots, y_{p}, \cdots, y_{p}^{\left(r_{1}-1\right)}\right]
$$

The expression of the new system after coordinate transformation is

$$
\begin{aligned}
& \dot{\xi}=\widehat{\mathrm{A}}_{1} \xi+\widehat{\mathrm{A}}_{2} \eta+\widehat{\mathrm{B}}_{1} \mathrm{u} \\
& \dot{\eta}=\widehat{\mathrm{A}}_{3} \xi+\widehat{\mathrm{A}}_{4} \eta+\widehat{\mathrm{B}}_{3} \mathrm{u}
\end{aligned}
$$

Where

$$
\widehat{\mathrm{A}}=\left[\begin{array}{ll}
\widehat{\mathrm{A}}_{1} & \widehat{\mathrm{A}}_{2} \\
\widehat{\mathrm{A}}_{3} & \widehat{\mathrm{A}}_{4}
\end{array}\right]=\mathrm{T}^{-1} \mathrm{AT} \text { and } \widehat{\mathrm{B}}=\left[\begin{array}{l}
\widehat{\mathrm{B}}_{1} \\
\widehat{\mathrm{B}}_{3}
\end{array}\right]
$$

The control law to maintain the exact tracking can be written

As

$$
u_{f f}=B_{y}^{-1}\left[y_{d}^{r}-A_{\xi} \xi(t)-A_{\eta} \eta(t)\right]
$$

Where

$$
\left[A_{\xi} A_{\eta}\right]=A_{x} T
$$

Also the internal dynamics

$$
\begin{aligned}
\dot{\eta} & =\widehat{A}_{3} \xi+\widehat{A}_{4} \eta+\widehat{B}_{2} B_{y}^{-1}\left[y_{d}^{r}-A_{\xi} \xi(t)-A_{\eta} \eta(t)\right] \\
& =\widehat{A}_{\eta} \eta(t)+\widehat{B}_{\eta} Y
\end{aligned}
$$

Where 
Waweru et al: Two-degree-of-freedom control of a multilink flexible manipulator using filtered inverse feedforward controller and strain feedback controller

$$
\begin{aligned}
& \widehat{\mathrm{A}}_{\eta}=\widehat{\mathrm{A}}_{4}-\widehat{\mathrm{B}}_{2} \mathrm{~B}_{\mathrm{y}}^{-1} \mathrm{~A}_{\eta} \\
& \left.\widehat{\mathrm{B}}_{\eta}=\widehat{\mathrm{A}}_{3}-\widehat{\mathrm{B}}_{2} \mathrm{~B}_{\mathrm{y}}^{-1} \mathrm{~A}_{\xi}+\widehat{\mathrm{B}}_{2} \mathrm{~B}_{\mathrm{y}}^{-1}\right] \\
& \mathrm{Y}=\left[\begin{array}{ll}
\xi & \mathrm{y}_{\mathrm{d}}^{(\mathrm{r})}
\end{array}\right]^{\mathrm{T}}
\end{aligned}
$$

in the same respect, equation 3 can now be written as

$$
\begin{aligned}
u_{f f} & =B_{y}^{-1}\left[y_{d}^{(d)}-A_{\xi} \xi(t)-A_{\eta} \eta(t)\right] \\
& =-B_{y}^{-1} A_{\eta} \eta(t)-\left[B_{y}^{-1} A_{\xi}-B_{y}^{-1}\right] Y \\
& =C_{\eta} \eta(t)+D_{Y} Y(t)
\end{aligned}
$$

Where

$$
\begin{aligned}
& \mathrm{C}_{\eta}=-\mathrm{B}_{\mathrm{y}}^{-1} \mathrm{~A}_{\eta} \\
& \mathrm{D}_{\mathrm{Y}}=-\left[\mathrm{B}_{\mathrm{y}}^{-1} \mathrm{~A}_{\xi}-\mathrm{B}_{\mathrm{y}}^{-1}\right]
\end{aligned}
$$

Equation (7) together with equation (8) form the inverse system and can be represented in state space form as in Figure 4

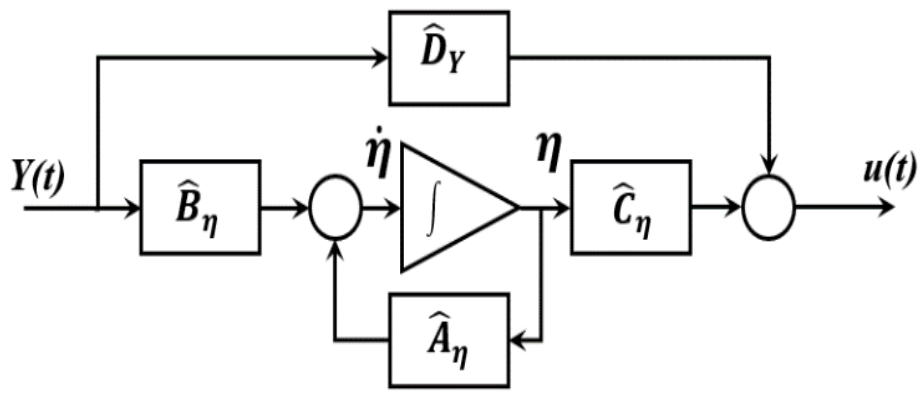

Figure 1: Block diagram of the inverse system

\section{INVERTING THE FLEXIBLE MANIPULATOR MODEL}

The model has 17 states as follows: 


\begin{tabular}{|c|c|}
\hline $\begin{aligned} & \text { - } x_{1}(t)=i_{1}(t) \\
& \text { - } x_{2}(t)=w_{1}(t) \\
& \text { - } x_{3}(t)=w_{1}(t) \\
& \text { - } x_{4}(t)=w_{2}(t) \\
& \text { - } x_{5}(t)=\dot{w}_{2}(t) \\
& \text { - } x_{6}(t)=w_{3}(t) \\
& \text { - } x_{7}(t)=\dot{w}_{3}(t) \\
& \text { - } x_{8}(t)=w_{4}(t) \\
& \text { - } x_{9}(t)=\dot{w}_{4}(t)\end{aligned}$ & $\begin{array}{l}\text { - } x_{10}(t)=\theta_{1}(t) \\
\text { - } x_{11}(t)=\dot{\theta}_{1}(t) \\
\text { - } x_{12}(t)=\theta_{2}(t) \\
\text { - } x_{13}(t)=\dot{\theta}_{2}(t) \\
\text { - } x_{14}(t)=\theta_{3}(t) \\
\text { - } x_{15}(t)=\dot{\theta}_{3}(t) \\
\text { - } x_{16}(t)=i_{3}(t) \\
\text { - } x_{17}(t)=i_{2}(t)\end{array}$ \\
\hline
\end{tabular}

Where $i_{j}$ denotes joint $j$ motor current, $\theta_{j}, \dot{\theta}_{j}$ angle and velocity of joint $j=1,2,3$ respectively whereas $\left(w_{1} ; w_{2}\right),\left(w_{3} ; w_{4}\right)$ and their derivatives denotes the flexure variable for links 1 and 2 respectively.

Having three inputs, three outputs and a relative degree of three $(r=3)$, the internal dynamics were taken as the flexure variables:

$$
\eta(\mathrm{t})=\left[\begin{array}{llllllll}
x_{2}(t) & x_{3}(t) & x_{4}(t) & x_{5}(t) & x_{6}(t) & x_{7}(t) & x_{8}(t) & x_{9}(t)
\end{array}\right]^{\mathrm{T}}
$$

Whereas the output and its derivatives were takes as the three joint angles and their derivatives.

Figure 5 below show the pole zero map of the plant and the inverse system.
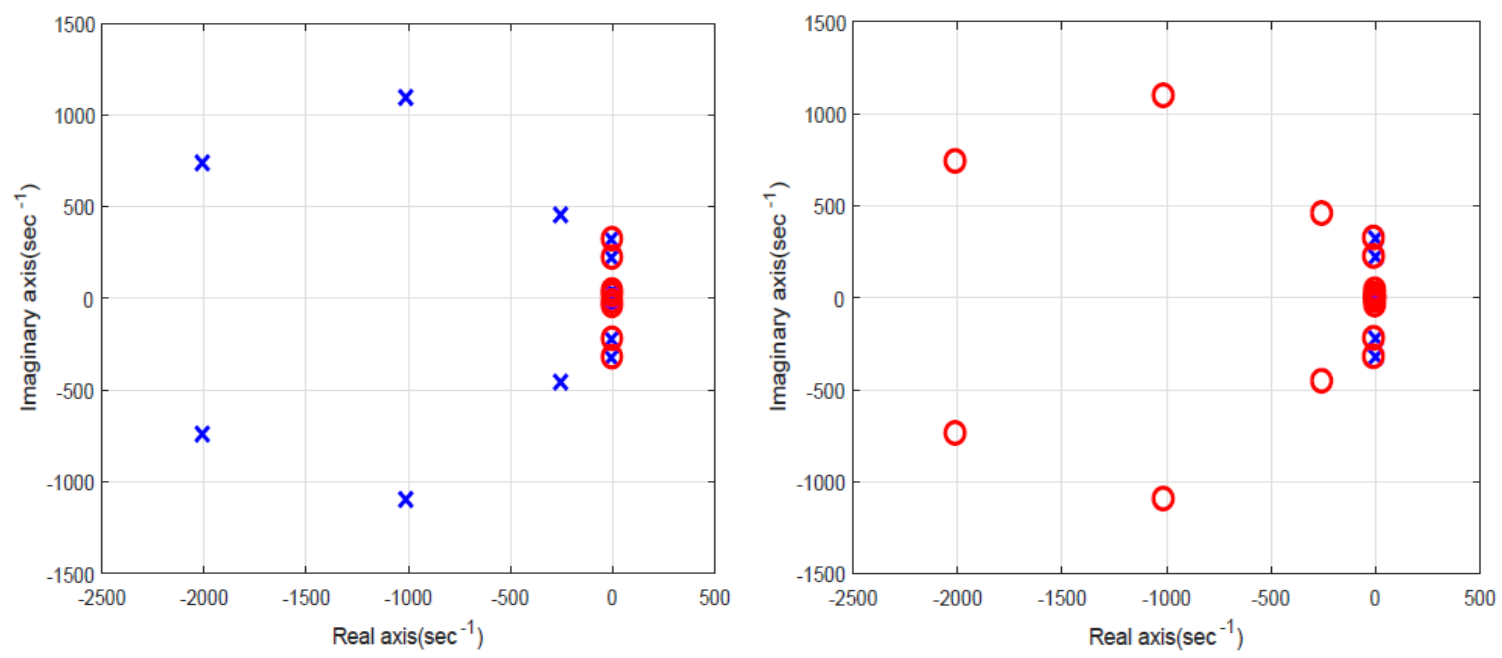


\section{Waweru et al: Two-degree-of-freedom control of a multilink flexible manipulator using filtered inverse feedforward controller and strain feedback controller}
(a). Pole-zero map of the plant
(b). Pole-zero map of the inverse

Figure 2: Poles and zeros of the plant and its inverse

The system was found to be neither state controllable nor observable. Also, from the figure, we can see that the system is non-hyperbolic i.e., we have poles on the imaginary axis. This has the negative implication that the poles of the system cannot be arbitrarily placed. However, it was found that after transformation, $(\mathrm{A} \eta ; \mathrm{B} \eta)$ is fully controllable and the poles in the subsystem involved with the internal dynamics (flexure variables) can be arbitrarily place.

Non-hyperbolicity is a situation where the poles of a dynamic system are located on the imaginary axis. Whereas the system is not unstable, this scenario is undesirable especially in vibration control as the internal dynamics, flexure variables in this case remains constant with time. To make the system hyperbolic, pole assignment method was employed in the internal

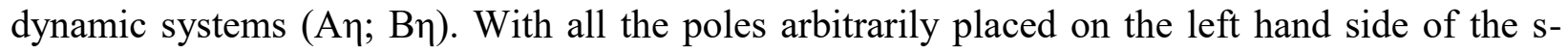
plane, the convergence of the internal dynamics is assured. The internal dynamics $\eta(t)$ can be solved conventionally by integrating forward in time as

$$
\eta(t)=\int_{-\infty}^{t} e^{A_{\eta}(t-\tau)} B_{\eta} Y_{d}(\tau) d \tau
$$

Since the system involves the derivatives of the desired output, it is required that this signal be differentiable up to degree $\mathrm{r}^{\mathrm{p}}$. This was solved by augmenting the inverse system with lowpass filter as in the internal model architecture (Zafiriou, 1989; Rivera, 1999) of the form

$$
f(s)=\frac{1}{(\lambda s+1)^{n}}
$$


The order of the filter $n$ is chosen large enough to make the inverse system proper. On the other hand, the adjustable parameter $\lambda$ determine the filter rolloff which in turn determines the speed of response. Increasing the values of $\lambda$ makes the response sluggish and vice versa.

\section{DIRECT STRAIN FEEDBACK}

Theory of Direct Strain Feedback (DSFB) developed by Luo in (Luo, 1993; Sakawa, 1993) and experimented with a one-link flexible manipulator. In this control scheme, the strain measured at the root of the flexible link is multiplied by constant gain $k$ and the resultant signal is used to modify the control law. In (Luo, 1993), the author shows that the technique can satisfactorily damp out link vibrations. He also analytically derived the prove that the resulting closed loop is asymptotically stable.

Block diagram showing the hybrid of the inverse feedforward controller and the DSFB is as shown in Figure 3. In the figure, the desired joint angles are fed into the inverse feedforward controller whose output is input to the plant to reproduce this desired angle. This means that very high and unsafe speeds are expected. To prevent this, desired angles are fed to the inverse feedforward controller through lowpass filters. Further suppression of vibrations is enhanced by measuring the link vibrations and feeding it back as in the figure.

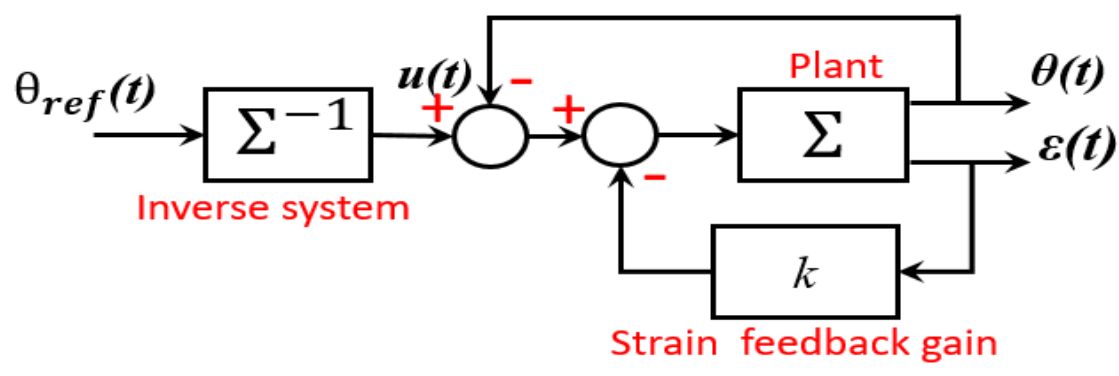


Waweru et al: Two-degree-of-freedom control of a multilink flexible manipulator using filtered inverse feedforward controller and strain feedback controller

Figure 3: Hybrid control configuration

\section{EXPERIMENTAL RESULTS AND DISCUSSION}

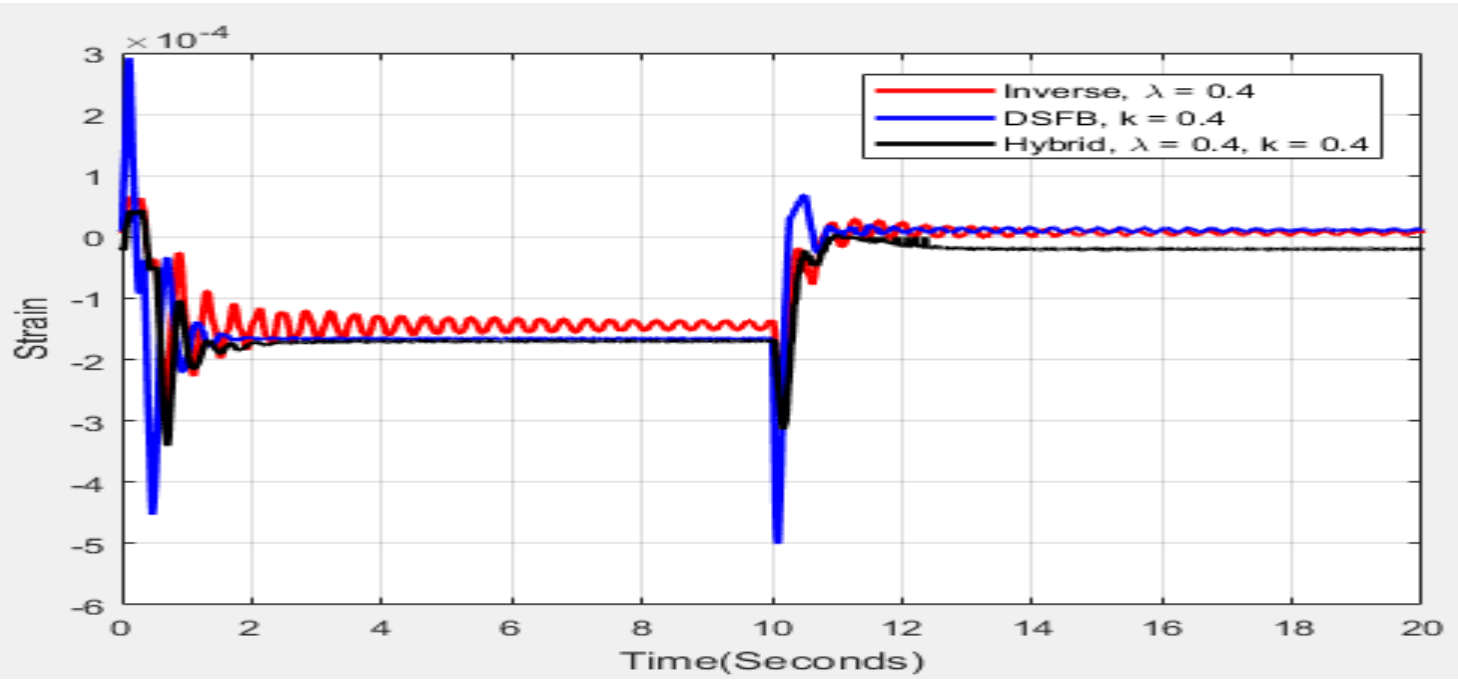

a) Link 1, in plane

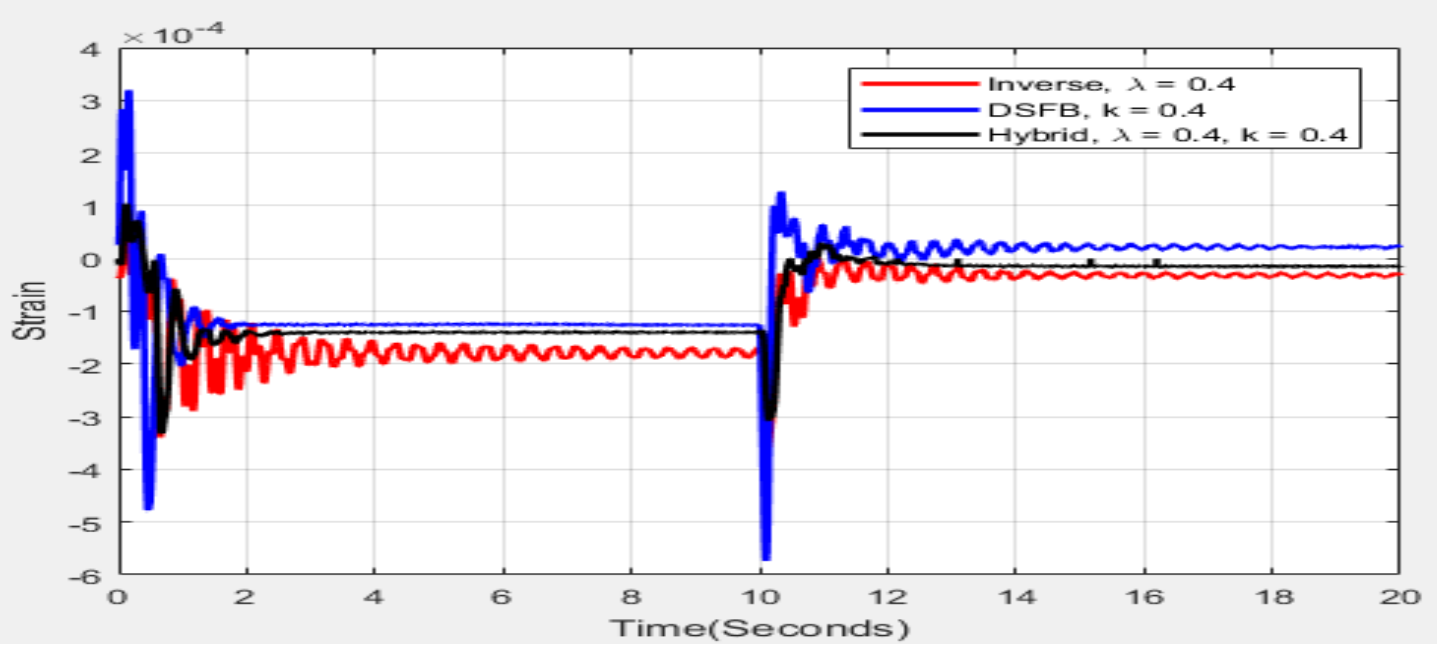


b) Link 2 in plane

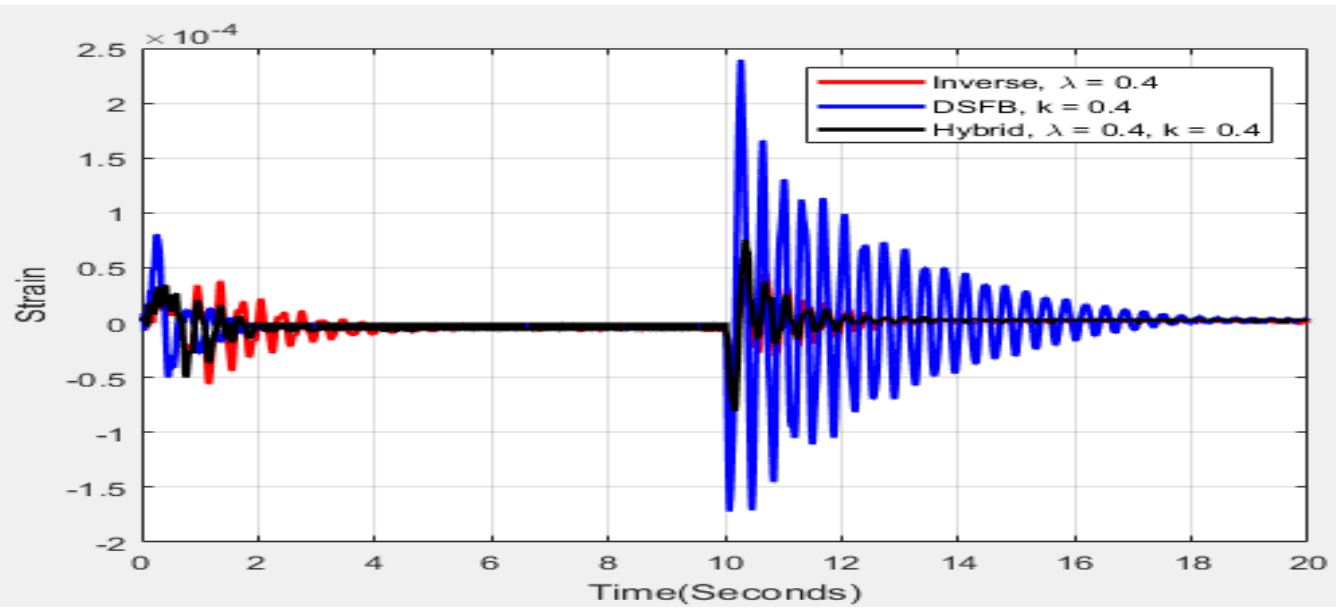

c) Link 1 torsion

Figure 4: Link vibration without load

Figure 4 shows the link strain for the three joints with filter time constant $\lambda=0.4$ for the inverse controller, strain feedback $\mathrm{k}=0.4$ for the strain feedback controller and without any load at the distal end of link 2. For the inverse controller, the link strain has a minimal amplitude but the response is characterized by residue vibration lasting for the entire period. On the other hand, strain feedback controller is characterized by very high initial strain values though it decays very fast. A hybrid of the two type of controllers yields a response bearing the strengths of individual controllers' i.e. minimal initial strain followed by residues which decays to zero even faster than that of strain feedback alone.

The improvement is evident from the strain power spectral densities shown in Figure 5. From the figures, there remain $3 \mathrm{~Hz}$ residue vibrations in the inverse controlled system. The individual 
Waweru et al: Two-degree-of-freedom control of a multilink flexible manipulator using filtered inverse feedforward controller and strain feedback controller

controllers dampen different frequency ranges with the hybrid performing better than the individual controllers.

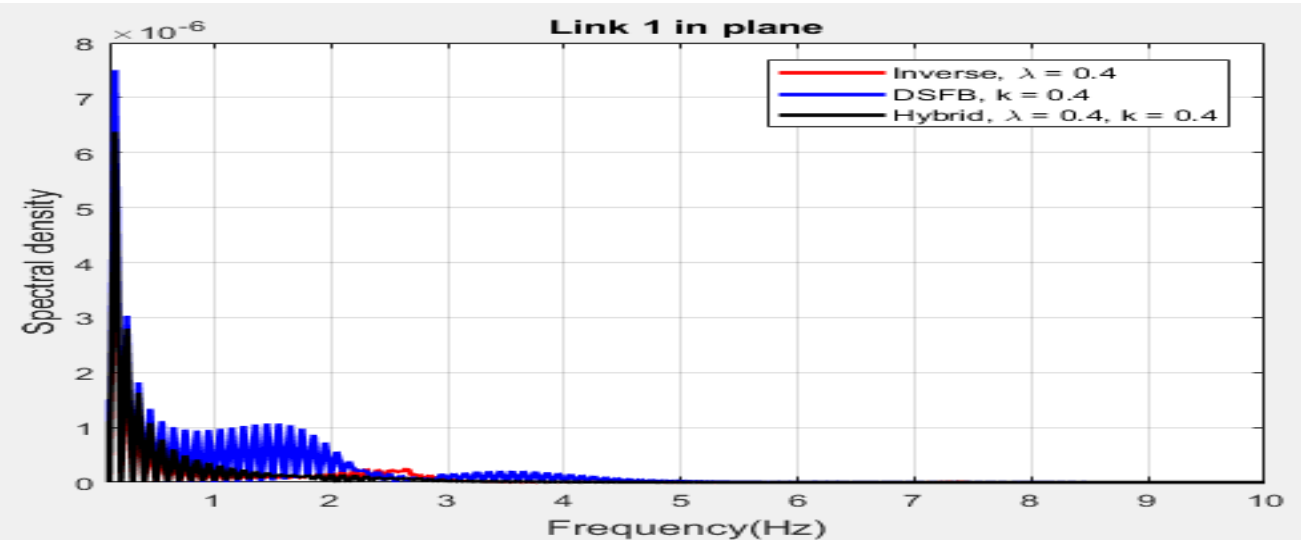

a) Link 1, in plane

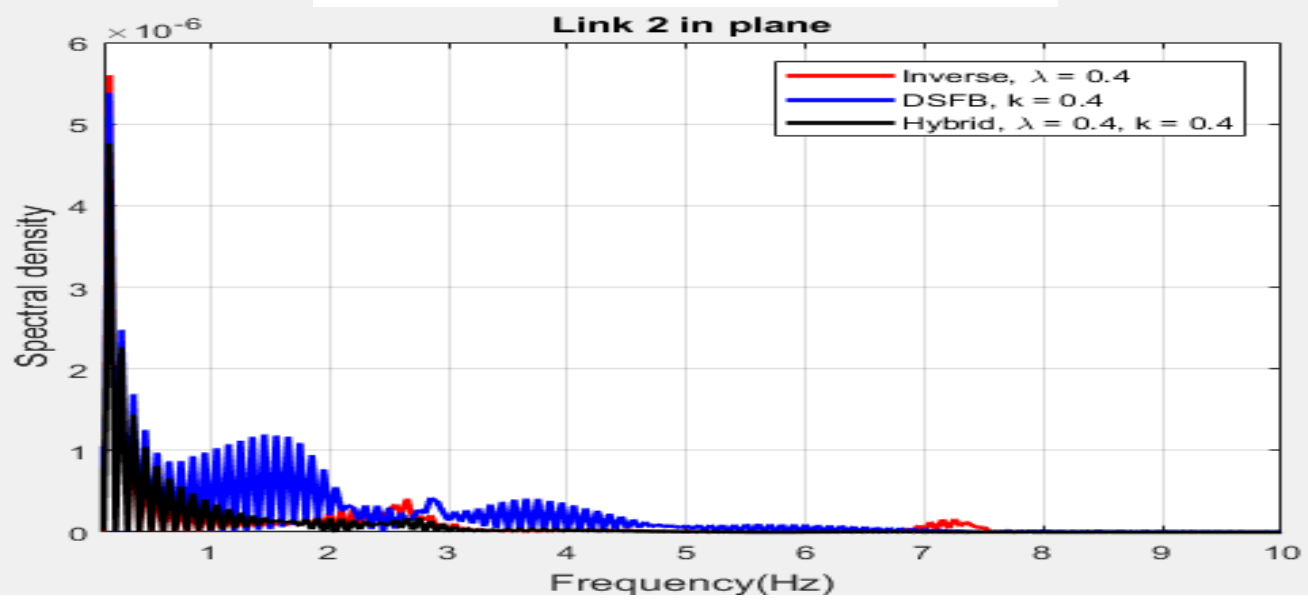

b) Link 2 in plane

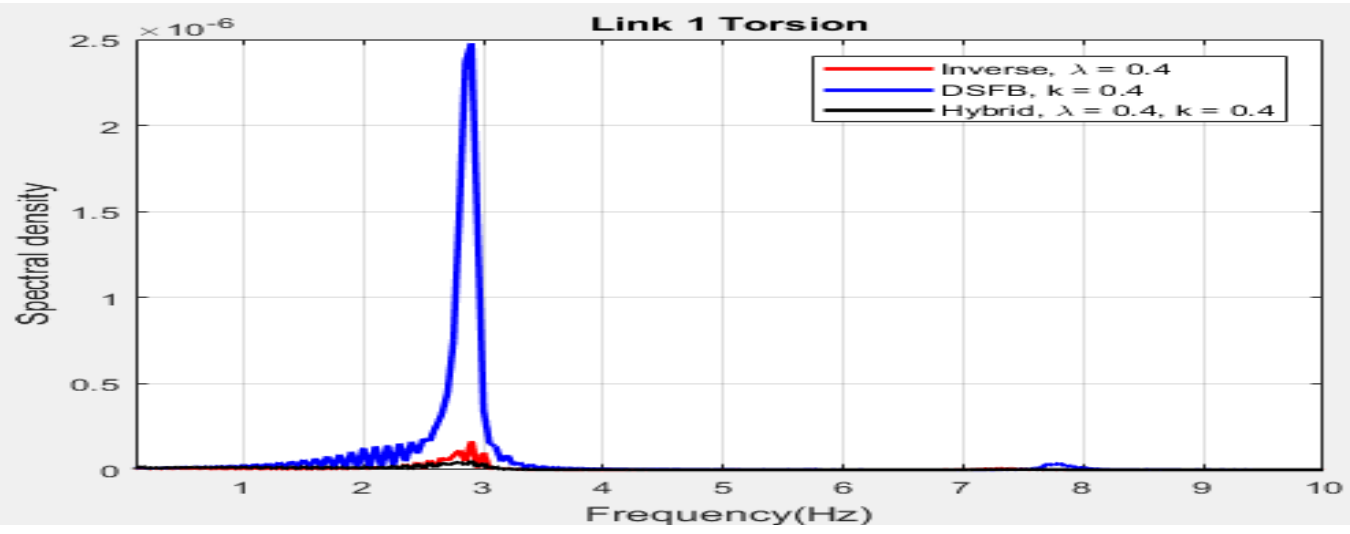

c) Link1 torsion 
Figure 5: Strain spectral power density without load

With a load of $100 \mathrm{~g}$ attached at the distal end of link 2, Figure 6 shows the link strain for links 1 and 2(in plane and torsion). From the figure, vibrations are a bit severe relative to those experience in a system without load. However, the same behaviour observed in the no load case applies to this case as well.

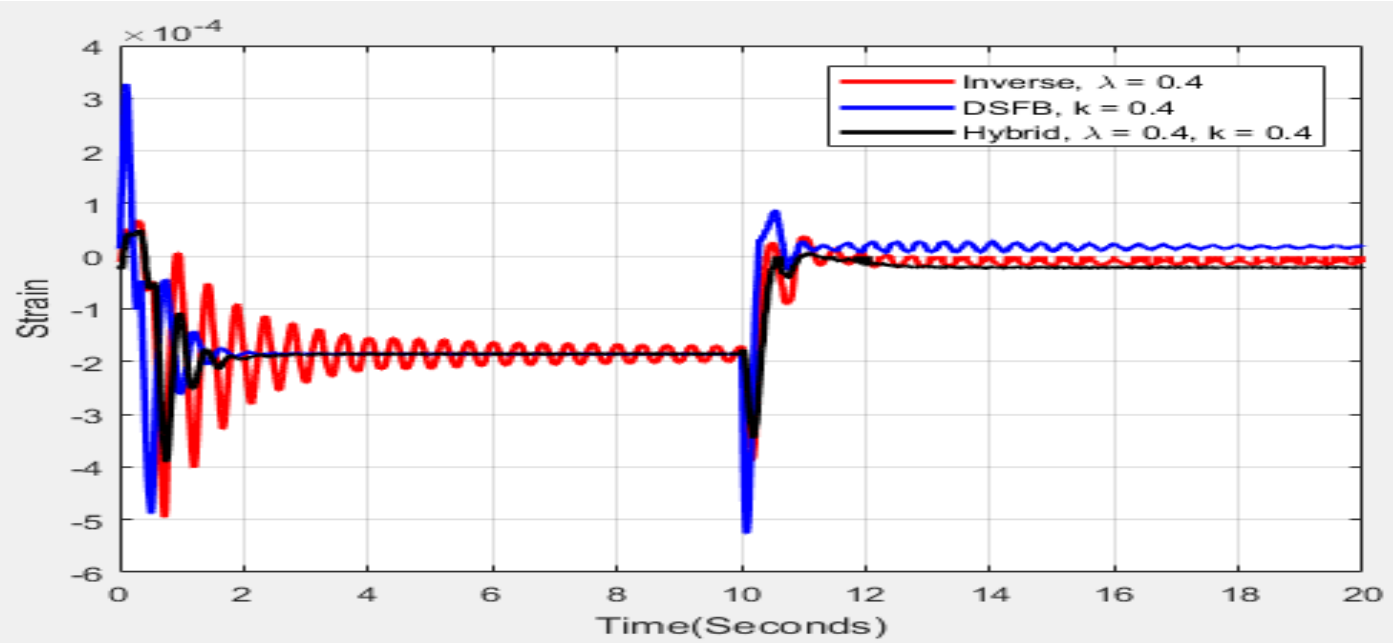

a) Link 1 in plane

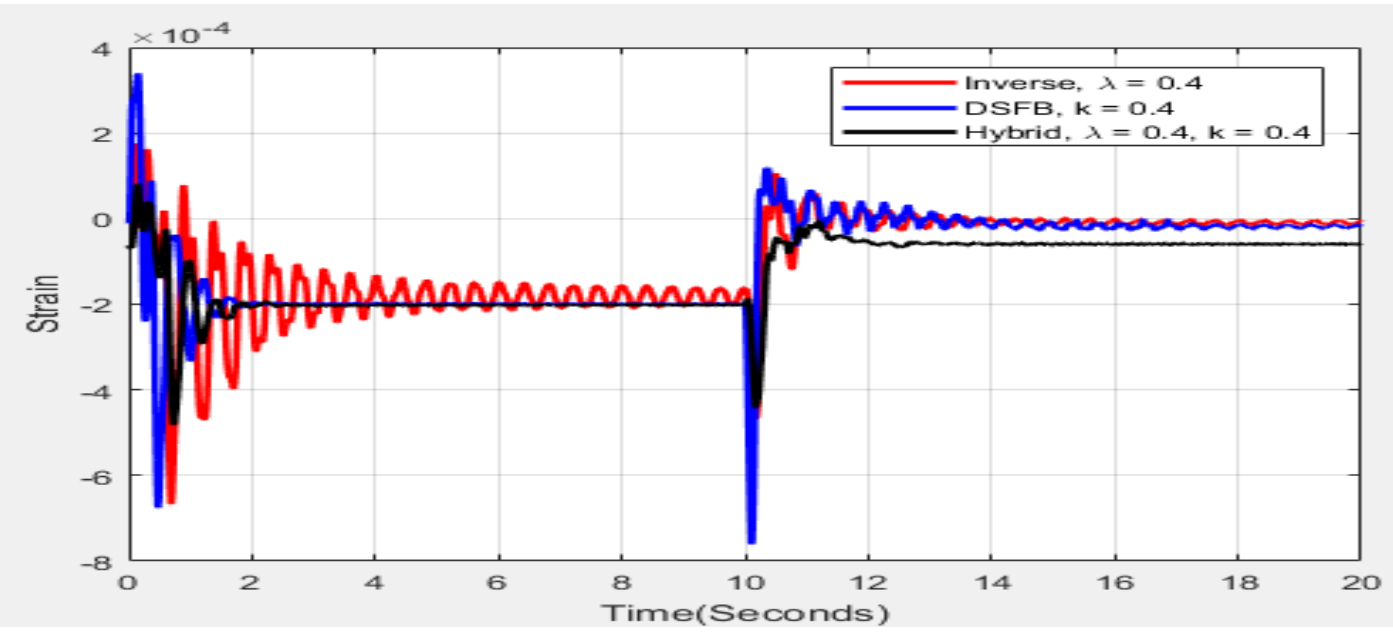

b) Link 2 in plane 
Waweru et al: Two-degree-of-freedom control of a multilink flexible manipulator using filtered inverse feedforward controller and strain feedback controller

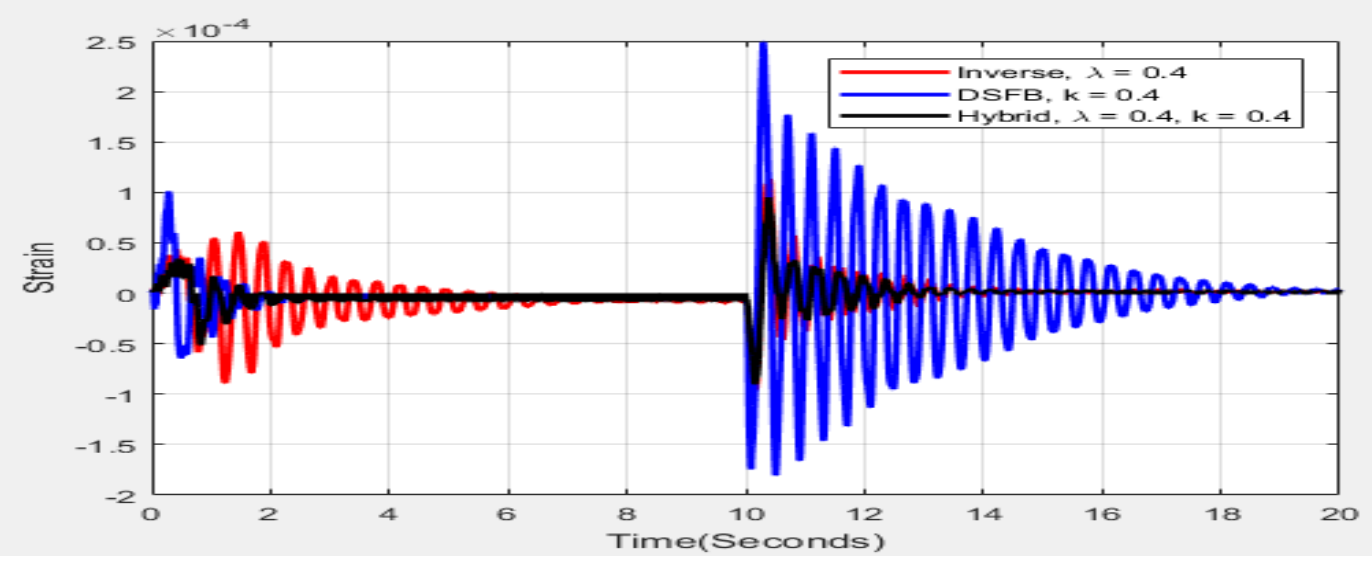

d) Link 1 torsion

Figure 6: Link vibration with a load of $100 \mathrm{~g}$

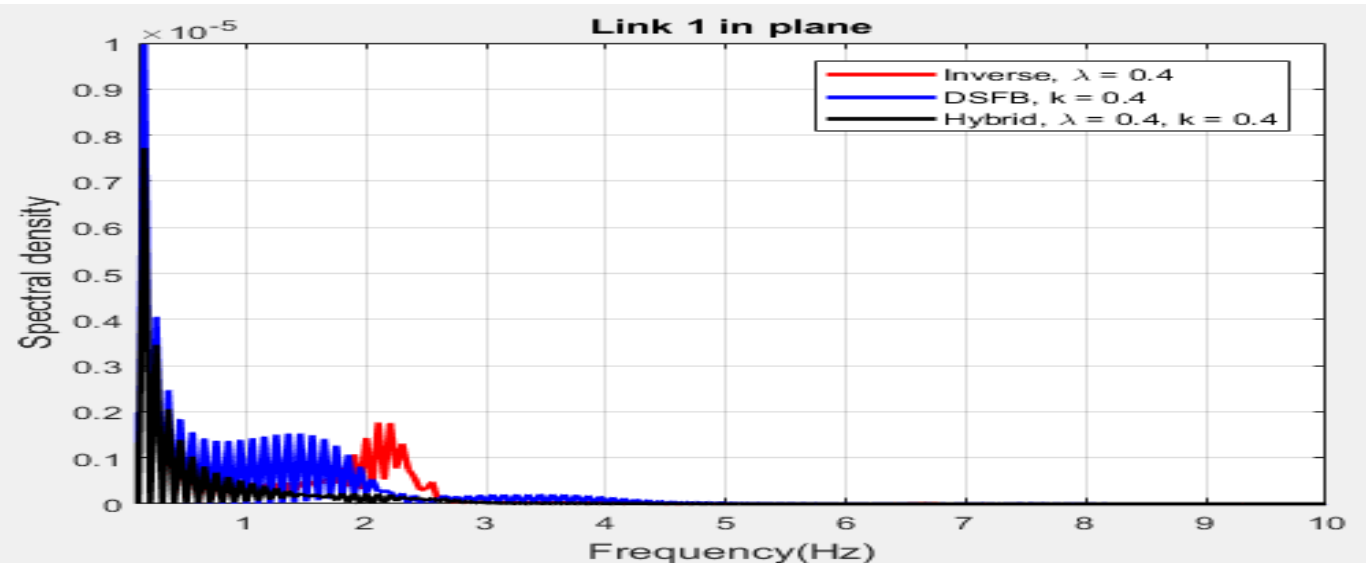

a) Link 1 in plane

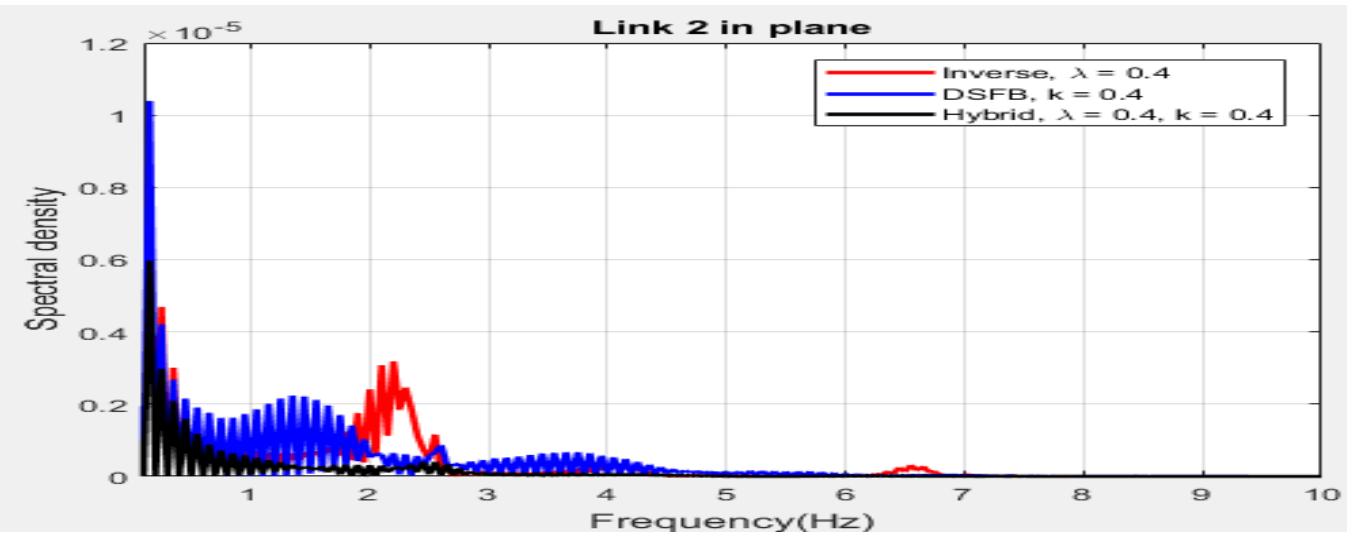


b) Link 2 in plane

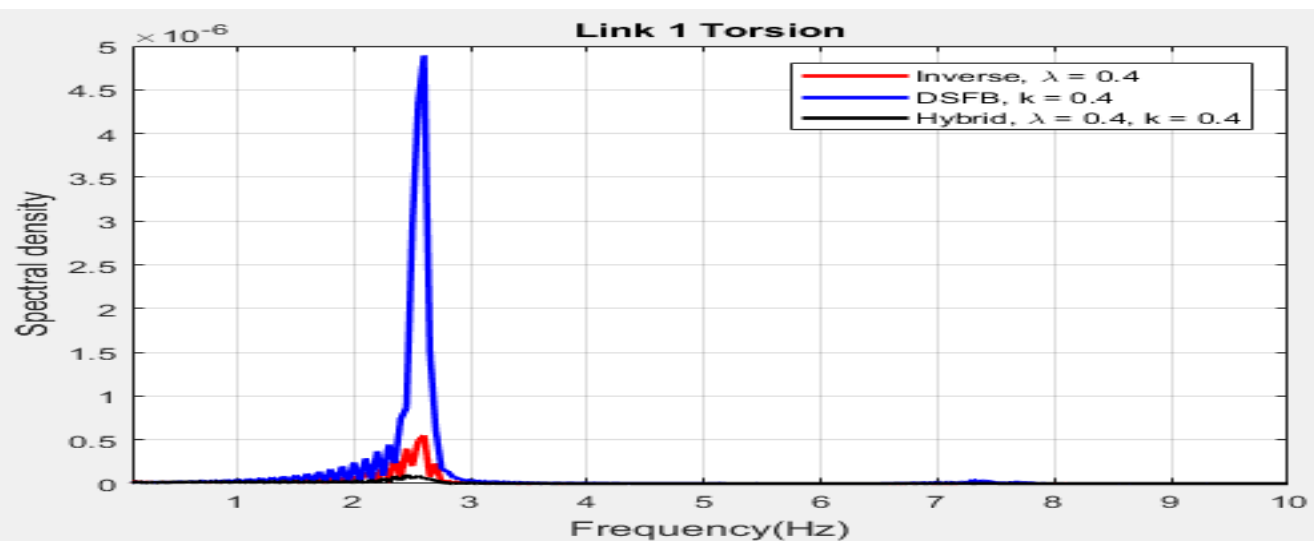

c) Link 1 torsion

Figure 7: Strain spectral power density with a load of $100 \mathrm{~g}$

\section{CONCLUSION}

In this paper, an inverse system for a multilink flexible manipulator is successfully developed and together with strain feedback used to mitigate link vibrations. The results presented shows that whereas inverse controller can reduce the amplitude of the vibrations, there are residue that last for the entire period. On the other hand, though strain feedback controller dampens vibration very fast, the amplitude at the outset is very high. The hybrid of the two yields not only minimal strain amplitudes but also shorten the time of the vibrations.

\section{BIBLIOGRAPHY}

Alhazza, Z. M. (2016). Multimode input shaping control of flexible robotic manipulators using frequency modulation. Jordan Journal of Mechanical \& Industrial Enginering, 10(3). 


\section{Waweru et al: Two-degree-of-freedom control of a multilink flexible manipulator using filtered inverse feedforward controller and strain feedback controller}

Luo, Z. H. (1993). Direct Strain Feedback Control of Flexible Robot Arms: New theoretical and experimental results. IEEE Transactions on Automatic Control, 38(11), 1610-1622.

Moheamani, S. O. (2003). A survey of recent innovations in vibrations damping and control using shunted piezoelectric transducers. IEEE Transactions in Control Systems, 482-494.

P.J.Moylan. (1977). Stable inversion of Linear systems. IEEE Transactions on Automatic Control, 74-78.

Rivera, D. E. (1999). Internal Model Control: A comprehensive Review. Tempe, Arizona: Arizona State University.

Sakawa, Z. H. (1993). Gain Adaptive direct strain feedback control of Flexible Robot Arms. TENCO '93 Proceedings, 1993 IEEE Region on Communication, Control and Power Engineering, 4, 199-202.

Sontag, D. L. (2002). Output-Input stability and Minimum-phase nonlinear systems. IEEE Transactions on Automatic Control, 47, 422-436.

Subudhi, K. L. (2016). A review on two-link flexible manipulators. Annual Review in Control, 42, 346-367.

Zafiriou, M. M. (1989). Robust process Control. Englewood Cliffs: Prentice Hall. 